Publié dans : Gilbert Buti, Anne Carol (dir.), Comportements, croyances et mémoires. Europe méridionale $X V^{e}-X X^{e}$ s., Aix-en-Provence, Presses universitaires de Provence, 2007, p. 71-82.

\title{
Les humeurs du lecteur : manières de lire et hypocondrie savante à Florence au XVIII ${ }^{\mathrm{e}}$ siècle.
}

\author{
Emmanuelle CHAPRON \\ Université de Provence (Département d'histoire)- \\ Maison Méditerranéenne des Sciences de l'Homme (TELEMME) \\ echapron@yahoo.com
}

Des pieds glacés par le pavement des bibliothèques aux flatulences de l'érudit amarré à son bureau, des mélancolies du penseur aux sanglots d'Alfieri, le corps du lecteur de l'époque moderne est fortement mobilisé dans l'acte de lire. Les savants florentins, bavards et plaintifs, en ont laissé de nombreuses traces dans leurs écrits, correspondances et diaires : matière à dresser, suivant le vœu de Georges Perec, « quelque chose comme une économie de la lecture sous ses aspects ergologiques (physiologie, travail musculaire) et socio-écologiques (environnement spatio-temporel) $»^{1}$. Cette histoire du corps du lecteur est pourtant à peine ébauchée. Plus qu'aux écrits du for privé ou aux sources normatives (traités de médecine, manuels de lecture), elle s'est surtout attachée jusqu'à présent à l'iconographie de la lecture, qui dit autant les valeurs attachées au livre que la réalité des pratiques ${ }^{2}$.

Cette anthropologie de l'effort savant permet pourtant de reprendre différemment l'étude des comportements du lecteur. Elle ouvre en particulier une perspective nouvelle sur la question controversée de la «révolution de la lecture ». La thèse est connue, quoique souvent présentée de manière schématique. Entre 1750 et 1850, à la faveur de la multiplication des livres, s'imposerait une nouvelle manière de lire. A la lecture intensive et répétitive d'un petit nombre de textes, souvent faite à haute voix dans un cadre collectif, succéderait un nouveau modèle dominant, celui d'une pratique extensive, à la fois plus intime et moins sacralisée, passant rapidement d'un livre à l'autre sans s'imprégner de la leçon du texte $^{3}$. Depuis trente ans, sans revenir sur le fond de la thèse, d'importantes nuances ont été apportées et de nouvelles interprétations proposées ${ }^{4}$. Paradoxalement, les professions intellectuelles sont peu prises en compte dans ces travaux qui privilégient plutôt les lecteurs « ordinaires ». Tout au long de l'époque moderne, ces milieux lettrés sont le creuset de pratiques diverses et complexes, parfois contradictoires, irréductibles en tout cas à l'opposition schématique entre lecture «intensive » et «extensive ». La « révolution savante de la lecture » ne peut donc être interprétée comme la succession de deux modalités dont l'une tendrait à prendre le pas sur l'autre, mais plutôt comme l'évolution d'un système ancien

\footnotetext{
${ }^{1}$ G. Perec, «Lire : esquisse socio-physiologique », Esprit, $\mathrm{n}^{\circ} 453,1976$, p. 9-20, p. 10.

${ }^{2}$ E. Schön, Der Verlust der Sinnlichkeit oder die Verwandlungen des Lesens, Stuttgart, Klett-Cotta, 1987. T. Plebani, Il « genere dei libri ». Storie e rappresentazioni della lettura al femminile e al maschile tra Medioevo e età moderna, Milan, F. Angeli, 2001.

${ }^{3}$ La thèse est surtout associée aux travaux de R. Engelsing, Der Bürger als Leser. Lesergeschichte in Deutschland 1500-1800, Stuttgart, Metzler, 1974. D. Hall, «Introduction : the uses of literacy in New England, 1660-1850 », in W. Joyce et al. (éd.), Printing and Society in Early America, Worcester, American Antiquarian Society, 1982, p. 1-47.

${ }^{4}$ R. Chartier (dir.), Histoires de la lecture. Un bilan des recherches, Paris, IMEC, 1995.
} 
et articulé de comportements, de postures et d'imaginaires, que les mutations de la librairie et des fonctions sociales de la lecture viendraient accélérer à la fin du XVIII ${ }^{\mathrm{e}}$ siècle.

On se propose d'éprouver cette hypothèse à partir d'une source d'une exceptionnelle richesse, les Efemeridi du florentin Giuseppe Pelli Bencivenni. Il s'agit d'un diaire en 80 volumes et près de 40000 pages, rédigé quotidiennement entre 1759 et 1808 . L'intérêt de ce texte vient de ce que le fonctionnaire grand-ducal est à plusieurs égards un "lecteur forcé », envahi de livres et de papiers ${ }^{5}$. Secrétaire de la Pratica Segreta de Pistoia et Pontremoli de 1762 à 1775, directeur de la Galerie des Offices de 1775 à 1792, Pelli remplit également à partir de 1763 les fonctions de censeur grand-ducal. Lecteur insatiable et auteur fécond, il collabore à la rédaction des Novelle letterarie de Florence et laisse derrière lui au fil des ans, véritables châteaux en Espagne, d'innombrables projets littéraires. Destinés à un cercle amical et familial comme à l'hypothétique lecteur «de 1900 ou 1930 », les Efemeridi inscrivent l'acte de lecture dans un espace d'écriture qui a ses propres conventions et des finalités spécifiques. Toutes précautions prises, les notes de Giuseppe Pelli n'en permettent pas moins de saisir les configurations plurielles du lire chez un lettré du XVIII ${ }^{\mathrm{e}}$ siècle. Elles donnent à voir la lecture, non seulement comme un dispositif social et textuel, comme la rencontre « de manières de lire $(. .$.$) et des protocoles de lecture déposés dans l'objet lu { }^{6}$, mais également comme une disposition du corps, mobilisant l'œil, la main, la «machine » toute entière. Sous la plume de Pelli, lecteur mélancolique et hypocondriaque, observateur attentif de lui-même, la lecture s'inscrit dans une physiologie complexe où elle est à la fois poison et potion.

\section{DU TAVOLINO AUX RIVES DE L'ARNO : FIGURES DE LA LECTURE.}

De la rumeur quotidienne des Efemeridi se dégagent plusieurs figures de la lecture, distinctes par leurs configurations spatiales, temporelles et sociales. Comme le souligne Daniel Roche, le temps du labeur intellectuel est un temps plastique, conjuguant des régularités quotidiennes et annuelles à une certaine liberté dans l'organisation du travail quotidien ${ }^{7}$. A plusieurs reprises au cours de ses années d'écriture, Giuseppe Pelli décrit minutieusement le déroulement de ses journées ${ }^{8}$. Levé vers 9 heures, il consacre la matinée à des audiences, à des visites, au rituel de la cioccolata, aux sessions de la Pratica Segreta. Il déjeune vers 1 heure, lit la gazette, s'octroie parfois une sieste. Les activités studieuses, courrier, comptes, examen des procès l'attachent à son bureau une partie de l'après-midi. Rasé de près, il s'adonne ensuite aux pratiques de la sociabilité : promenade vers 5-6 heures, conversazione de 7 à 11 heures, théâtre parfois. La nuit se poursuit studieuse, solitaire, concentrée et silencieuse, avant que le sommeil ne l'emporte vers 2 heures après minuit. Les déclinaisons quotidiennes de l'activité de lecture sont donc nombreuses : le travail diurne sans cesse interrompu s'oppose au fil continu des lectures nocturnes, l'activité solitaire du studiolo à la sociabilité des salons et à la collégialité des administrations, les lectures du loisir à celles du travail - la frontière entre les deux s'avérant tout de même, dans le cas des professions

\footnotetext{
${ }^{5}$ L'expression est de B. Fabian, « Der Gelehrte als Leser », in H. G. Göpfert (éd.), Buch und Leser, Hambourg, Hauswedell, 1977, p. 48-87. Les Efemeridi sont conservés à la Biblioteca Nazionale Centrale de Florence (Nuovi Acquisti, 1050 I [1759-1773, 30 vol.] et II [1773-1808, 50 vol.]). La première série a été mise en ligne (http://ferrovia.bncf.firenze.sbn.it/pelli/it/progetto.html). Sur Pelli (1729-1808), R. Pasta, «Profilo di un lettore », Editoria e cultura nel Settecento, Florence, Olschki, 1997, p. 193-224 ; S. Capecchi, « Ritratto di Giuseppe Pelli Bencivenni », Studi italiani, 2004-2005, p. 59-99.

${ }^{6}$ R. Chartier, «Du livre au lire », in Id. (dir.), Pratiques de la lecture, Paris, Payot \& Rivages, 2003 ( $1^{\text {ère }}$ éd. 1985), p. 81-117, p. 82.

${ }^{7}$ D. Roche, «L'intellectuel au travail », Annales ESC, 1982, n 3, p. 465-480, p. 477.

${ }^{8}$ Il tient même dans la semaine du 18 au 25 septembre 1772 un «journal exact de sa vie », suivant la suggestion d'un article du Spectator d'Addison (vol. III, discours 64 dans l'édition de Paris, 1723) (BNCF, NA 1050 I, vol. XXX, p. 9-20).
} 
intellectuelles, assez floue. La réalité des pratiques reste pourtant délicate à saisir, car le geste d'écriture agrège à la description du quotidien tout un imaginaire de la lecture. Ces nuits studieuses complaisamment évoquées sont autant un moment essentiel de la journée de travail qu'un aspect récurrent de la représentation des professions lettrées. Topos des éloges académiques et du discours médical consacré aux savants, c'est aussi un sujet de conversation pour Pelli et ses amis qui se plaisent à comparer leurs rythmes de travail et forgent autour de comportements partagés une identité sociale commune.

L'essentiel des activités intellectuelles diurnes ou nocturnes se joue dans un espace resserré, celui du studiolo domestique. C'est assis à son bureau que Pelli examine à la lumière de Balde et de Bartolo les très ennuyeux dossiers des procès criminels, mais aussi qu'il s'adonne à la plus légère lecture de la Zazirocratie de Tiphaigne de la Roche. Il y a certes de nombreuses façons de lire au bureau : assis face à la table ou de côté, le livre posé sur la table, tenu des deux mains ou d'une seule, la tête appuyée sur une main ou encore les deux coudes posés sur la table. Les Efemeridi ne permettent malheureusement pas d'identifier ces différentes postures, de même qu'ils taisent peut-être des configurations plus insolites - on sait que Cujas lisait volontiers allongé par terre, ses livres autour de lui - ou moins avouables. A rebours des représentations du XVIII ${ }^{\mathrm{e}}$ siècle qui peignent le lecteur ou la lectrice alanguie dans une confortable bergère, assis dans un café, voire sur un pas de porte, le journal de Pelli révèle en tout cas la grande continuité des postures mentales et physiques du lire dans les milieux lettrés.

A cette position assise sont associées deux autres caractéristiques de la posturologie savante de la lecture. En premier lieu, la position du lecteur est également celle d'un scripteur. Appliquée à extraire, à annoter, à peser, à confronter, à collationner, la lecture savante est faite la plume à la main, même dans le cas d'ouvrages aussi légers que la Zazirocratie, dont Pelli livre un compte-rendu fort minutieux. De l'omniprésence de la plume, l'objet livre porte la trace. Pelli n'hésite pas à annoter ses ouvrages et défend l'art de l'apostille : ses marginalia doivent conjurer les faussetés séductrices des auteurs modernes et le jugement qu'il appose sur la première page du livre, à la manière de Montaigne, constituer un aide-mémoire utile? Le second appendice du lecteur savant est son bureau, petit espace stratégique où cohabitent et s'organisent les différentes manières de lire, où s'inventent des rituels. Une sorte de géographie affective du tavolino se dégage des notes de Pelli, particulièrement sensible les jours où la neige et le grand vent de novembre le retiennent chez lui. Les dossiers de procès y forment un encombrement dont la vue lui soulève le cœur et dont il s'efforce chaque jour de «nettoyer » son bureau, afin «qu'ils n'y dorment pas »; la disparition des dossiers, aussitôt remplacés par d'autres, marque physiquement l'avancée du travail. A portée de main, les livres à peine reçus : comme à Montaigne, les voir et les feuilleter suffit à son plaisir pour « au moins 3 ou 4 jours », avant qu'il ne leur consacre les derniers instants de sa soirée, en $\mathrm{s}^{\prime}$ « enlevant le sommeil des yeux ${ }^{10}$. A côté de lui enfin, une «bibliothèque de table » partie réelle, partie imaginaire : les Essais de Montaigne, le traité de Tissot - on y reviendra.

\footnotetext{
${ }^{9}$ Il annote ainsi le Saggio sopra la politica e la legislazione romana de G. Bottone (s.l., 1772), «parce que l'auteur a du talent, mais soutient des choses fausses, ou hasardées avec enthousiasme, et une chaleur séductrice » (BNCF, NA $1050 \mathrm{I}$, vol. XXX, p. 70, 20 novembre 1772). Les notes manuscrites qu'il trouve sur les livres qu'il achète ne le gênent pas non plus : «Je ne suis pas très jaloux des livres. Ils doivent servir à leurs patrons, qui ont tout le droit de les tâcher en écrivant dans la marge ce qu'ils veulent pour leur commodité. Celui qui en prend trop soin ressemble à celui qui ne sort pas de chez lui quand il pleut pour ne pas abîmer ses chaussures. Il est vrai qu'ils perdent de leur valeur s'il n'ont pas servi à quelque lettré de premier ordre, mais les vêtements aussi coûtent moins cher lorsqu'ils ont déjà été portés. Est-ce une raison pour les laisser dans la garderobe ? » (ibid., vol. I, p. 189, 26 novembre 1759).

${ }_{10}$ Ibid., vol. IX, p. 127 ( $1^{\text {er }}$ avril 1763), citant le livre III, chapitre 3 des Essais : «Toutefois il se passera plusieurs jours, et des mois, sans que je les emploie : Ce sera tantôt, dis-je, ou demain, ou quand il me plaira ».
} 
Cet espace de travail est saturé d'imaginaire. Studiolo, stanzino, scrittoio, gabinetto : la diversité des dénominations, qui ouvrent sur d'autres champs sémantiques, n'affecte pas la profonde cohérence de cette représentation ${ }^{11}$. C'est d'abord le lieu de la distance avec le monde, qui permet l'observation et la théorisation, l'espace clos où s'élabore la pensée à la rencontre de quelques auteurs choisis. Le cabinet imaginaire de Pelli est une bibliothèque idéale, la «bibliothèque du philosophe honnête », bien distincte du fonds de livres professionnels. L' « autoportrait en cabinet » qu'il esquisse en mai 1770 s'inscrit certes dans une tradition iconographique éprouvée, mais il reconduit surtout l'image classique du studiolo comme locus amoenus, lieu propre à recréer l'esprit et à réjouir les sens autant que représentation d'un paysage intérieur, miroir intime de l'individu ${ }^{12}$.

\begin{abstract}
Je n'ai jamais fait mon portrait... Si un jour je veux, ou peux me le faire, je veux qu'il soit ainsi. Un demi-personnage assis dans un cabinet orné de portraits d'hommes illustres florentins avec leurs noms, avec quelques vases de fleurs et d'herbes odoriférantes. Le personnage devra porter une perruque et un habit long d'intérieur, mais noble. Qu'il y ait sur une table devant lui des livres épars, sur les tranches desquels on lise Pindare, Horace, Dante, Essais de Montaigne, Pope, Montesquieu. Que le personnage apparaisse sur le point d'écrire une lettre au marquis Cesare Beccheria Bonesana Milan, qu'il ait a près de lui une chienne bolonaise qui le caresse ${ }^{13}$.
\end{abstract}

Préféré aux divertissements publics et au théâtre, le cabinet est enfin le lieu de la «parfaite solitude ", l'endroit où s'éprouve la paix de l'âme : ne pas pouvoir, ou ne pas vouloir s'y appliquer «signifie que l'esprit n'est pas en calme, ni dominé par lui-même, mais par quelque passion ${ }^{14}$.

La relation étroite qui se tisse dans les Efemeridi entre lecture et écriture accentue exagérément l'impression d'un lire reclus entre les parois du cabinet, dans l'espace du for privé. Le silencieux dialogue avec les textes est en réalité inséparable des conversations de salon, de travail, de théâtre ou de promenade, comme des visites chez les libraires ou dans les bibliothèques. Comme les salons parisiens, la conversazione et le crocchio (cercle) florentins sont le lieu de lectures collectives, que l'on retrouve à l'automne et au printemps dans les villégiatures de campagne. Elles peuvent être lecture à haute voix, comme d'une lettre de Frédéric II au marquis d'Argens, entendue par Pelli une matinée d'octobre $1760 \mathrm{chez}$ le père Adami, ou lecture ensemble, comme dans la villa de Maiano de l'abbé Gianni, où l'on passe les soirées à lire, discuter, jaser et échafauder des projets de réforme ${ }^{15}$. La lecture partagée alimente l'échange savant, mondain ou politique, à un moment où la question des réformes échappe, dans un mouvement partiellement contrôlé par le prince, aux arcana imperii, devenant matière à réflexion des élites florentines.

Les séjours à la campagne, les promenades sous les frondaisons sauvages du parc de Boboli ou les marches le long de l'Arno, au-delà des portes de la ville, sont également pour Pelli l'occasion de lectures solitaires. Il est difficile d'apprécier ce que disent ces mentions éparses des pratiques réelles du lecteur, de leur fréquence, de l'incidence du grand air sur la

\footnotetext{
${ }^{11}$ Le gabinetto (89 occurrences) est fortement polarisé par le monde des collections (41 occurrences en ce sens), dans une moindre mesure par la sphère politique. Sur le studiolo (5 occurrences), W. Liebenwein, Studiolo. Storia e tipologia di uno spazio culturale, Ferrare, Panini, 1988, rééd. 2005 (1 $1^{\text {ere }}$ éd. all. 1977).

${ }^{12}$ B. Beugnot, «L'ermitage parmi les livres : Image de la bibliothèque classique », Revue française d'histoire du livre, 24, 1979, p. 687-707. «Ce n'est pas une mauvaise règle, pour connaitre le fond des personnes, de voir quels livres elles tiennent dans leur cabinet, lesquelles elles lisent le plus souvent, lesquelles enfin elles louent le plus » (BNCF, NA 1050 I, vol. III, p. 161, 13 novembre 1760)

${ }^{13}$ Ibid., vol. XXVI, p. 15-16, 26 mai 1770. Cesare Beccaria est l'auteur du traité Dei delitti e delle pene, Munich [Florence], Bonducci, 1764.

${ }^{14}$ Ibid., vol. XVI, p. 150

15 Ibid., vol. III, p. 113 (5 octobre 1760), vol. XV, p. 105 (7 novembre 1765). M. Madignier, Sociabilité informelle et pratiques sociales en Italie: les salons romains et florentins au XVIII siècle, thèse de l'Institut universitaire européen, dir. D. Roche, 1999.
} 
manière de lire, et ce qu'elles doivent à une imagerie déjà ancienne, renouvelée par la sensibilité préromantique. Présent depuis le Moyen Age, le motif de la lecture en plein air s'est développé sous la Renaissance avec la multiplication des ouvrages profanes en petit format, comme ces poètes amoureux que Machiavel s'en va lire près de la fontaine. A la fin du XVIII ${ }^{\mathrm{e}}$ siècle, dans une nature esthétisée, le motif envahit les représentations littéraires et figurées de la lecture. Les pratiques bucoliques de Giuseppe Pelli sont en réalité fort éloignées des rêveries du promeneur solitaire comme des affres des lecteurs de Werther. Assez peu réceptif à son environnement sensible, Pelli tend à recréer hors du studiolo les conditions d'une lecture studieuse : «J'aime la solitude, écrit-il en novembre 1759, mais elle m'ennuie si je lâche la bride à mon esprit. C'est pour cela que j'arrive mieux à me divertir en marchant avec un livre à la main, parce que je force mon âme à penser à ma lecture, et non à ce que lui dicte le caprice ». Les lectures qu'il convie en villégiature s'inscrivent également dans un registre éprouvé : comme Madame de Sévigné aux Rochers, Pelli emporte presque toujours à la campagne des livres d'histoire, car c'est pour lui « un plaisir très sensible de réfléchir, dans cette solitude et dans ce loisir placide, aux vicissitudes humaines $»^{16}$.

\section{PHYSIOLOGIE DE LA LECTURE.}

Les contraintes physiques et mentales associées à la lecture et au travail intellectuel ont suscité un discours médical spécifique. Entre le $\mathrm{XVI}^{\mathrm{e}}$ siècle et le milieu du XIX ${ }^{\mathrm{e}}$ siècle, une trentaine de traités rassemblent les lieux communs sur l'hygiène et les pathologies des gens de lettres. Giuseppe Pelli est un exemple, sans doute fort ordinaire, de la manière dont les savants ont pratiqué et assimilé cette littérature. Comme son contemporain Angelo Maria Bandini, le bibliothécaire florentin, il a lu le traité de Tissot De la santé des gens de lettres (1768) «avec beaucoup de plaisir, et avec l'espérance d'en tirer profit », mais aussi le traité De vita sana, seu De cura valetudinis eorum qui litterarum studio incumbunt de Marsile Ficin (1501) (dont il juge les remèdes, fondés sur les principes de la médecine astrologique, de peu d'utilité), le traité De tuenda sanitate studiosorum et literatorum de Gregor Horst (1617), la dissertation De studiis per regulas diaeteticas facilitandis et prolonganda litteratorum vita de Friedrich Hoffmann (1697), le discours De literatorum morbis de Bernardino Ramazzini (1700), enfin l'ouvrage de Giuseppe Antonio Pujati, Della preservazione della salute dei letterati $(1762)^{17}$. Tous pointent dans « les travaux assidus de l'esprit, \& le continuel repos du corps » la source des maux infinis dont sont affligés les lettrés. Pelli ne peut ignorer que ses lectures, faisant affluer le sang au cerveau, sont risque d'apoplexie, de tumeurs, d'insomnies et de maux de tête, que sa vie sédentaire ralentit le mouvement de son sang, dérange ses intestins, favorise l'accumulation des humeurs excrémentielles, et que la conjonction des deux le pousse insensiblement vers l'issue fatale.

De fait, quoique Pelli se vante de jouir d'une bonne santé, il fait de ses Efemeridi l'intarissable récit de ses tourments physiques et psychologiques. Les affections de l'hypocondre qui l'assaillent régulièrement, sous la forme de troubles digestifs (flatulences, aigreurs d'estomac, coliques) ou de douleurs de poitrine (élancements, catarrhe, palpitations), y sont moins pénibles que ces grandes inquiétudes, cette tristesse d'âme, cette sombre mélancolie qui l'étreignent parfois pendant plusieurs jours, l'empêchant de travailler et de se mêler au monde. Si Pelli reconduit dans ses grands traits la figure mélancolique et hypocondriaque que les médecins prêtent aux gens de lettres depuis Hippocrate et Galien, il la recompose pourtant à sa manière.

\footnotetext{
${ }^{16}$ BNCF, NA 1050 I, vol. I, p. 178 (19 novembre 1759) et vol. XII, p. 66 (4 juin 1764).

17 Ibid., vol. XXII, p. 18, 12 août 1768 : «il est utile pour qui a déterminé de vivre dans les études et l'application d'avoir ce petit livre [le traité de Tissot] sur son bureau ».
} 
Sujet à de douloureuses flatulences, il prête une extrême attention aux méfaits de la sédentarité, réputée provoquer des troubles digestifs. La parade consiste en quelques règles d'hygiène déjà mises en place en 1759 : supprimer le repas du soir pour pouvoir s'appliquer sans incommodité aux travaux nocturnes, ne pas se remettre au tavolino immédiatement après le déjeuner et interrompre quotidiennement l'étude par l'exercice de la marche. La lecture de Tissot, qu'il annote soigneusement en 1768, ne modifie pas ses pratiques, quoique le médecin suisse condamne tout ensemble le travail de nuit et l'usage de la sieste. Le changement est ailleurs, dans la manière de comprendre l'effet de la sédentarité sur la santé du lecteur. A partir de 1765 apparaît dans les Efemeridi tout un nouveau vocabulaire fibrillaire. Sans détrôner la conception humorale, il témoigne de l'influence des travaux de Haller sur l'irritabilité, et plus largement des mutations dans la représentation du fonctionnement corporel, intégrant les principes de tension et d'excitation. L'objet de la promenade de Pelli est alors de rendre leur «ton » aux fibres et d'acquérir un "meilleur degré d'élasticité musculaire », pour combattre cette rigidité que la vie sédentaire donne aux fibres et qui rend l'homme indolent. La marche, exercice hygiénique séculaire, prend des airs de «médecine gymnastique ${ }^{18}$. La conception fibrillaire permet même de résoudre l'un des plus douloureux mystères du monde savant, celui des variations quotidiennes de la vivacité de l'esprit, en les rapportant à l'état de tension des fibres et des nerfs ${ }^{19}$.

Des maux du corps à ceux de l'esprit, à la sombre mélancolie, le discours médical croise d'autres représentations. Les tergiversations de la théorie médicale ne passent pas : l'humeur noire de Pelli a à faire avec l'hypocondre, mais le rôle du sang ne lui semble pas clair et les nerfs n'y participent pas encore. Fille des humanistes, la mélancolie est aussi pour le diariste cette délectation morose, cette triste joie qui accompagne les progrès de la conscience de soi et le goût de la solitude ${ }^{20}$. Sentiment d'imperfection, «née de ne pas être comme je voudrais », elle croise enfin l'inquiétude des Lumières, celle qui pousse l'esprit à agir ${ }^{21}$. Pelli fait en effet de ses innombrables activités intellectuelles un refuge efficace contre les idées noires. «Antidote contre les douleurs du corps et de l'esprit»sont les livres qui font rire, tandis que d'autres provoquent, dans l'intensité de leur lecture, une véritable catharsis.

La lecture des œuvres de d'Alembert, l'homme le plus grand que je connaisse en ce siècle, m'agrandit l'esprit, nourrit mon entendement, me réjouit le cœur par le plaisir sensible qu'il m'apporte doucement (...) A lire les choses de monsieur Thomas, mon cœur s'agrandit, je sens une jouissance suave dans mon esprit, et je connais tout l'empire de la vérité, et de la vertu. ${ }^{22}$

\footnotetext{
${ }^{18}$ Ibid., vol. XIV, p. 117 (18 juin 1765) et vol. XXVII, p. 135 (3 mai 1771). « Je suis plus porté à donner foi à la médecine gymnastique qu'autres méthodes pour soigner les maladies », écrit-il après lecture du traité de G. Benvenuti sur les effets du mouvement à cheval (Lucques, Giusti, 1760) (ibid., vol. VII, p. 11, 13 février 1762).

${ }^{19}$ Pelli cite en ce sens le traité Della rapidità dell'idee de J. Belgrado (Modène, Montanari, 1770) évoquant « les jours sereins et gais, où les fibres sont plus tendues, les muscles élastiques, les esprits plus agiles et plus vifs » (ibid., vol. XXVI, p. 73, 22 juillet 1770). Sur le «prestige de la fibre » dans la seconde moitié du XVIII ${ }^{\mathrm{e}}$ siècle, G. Vigarello (dir.), Histoire du corps, 1. De la Renaissance aux Lumières, Paris, Seuil, 2005, p. $293-294$ et p. 362-365.

${ }^{20}$ « Ma mélancolie à écrire ces Efemeridi est un effet du plaisir que je prends à la solitude » (BNCF, NA $1050 \mathrm{I}$, vol. X, p. 102, 27 juillet 1763).

${ }^{21}$ Ibid., vol. XI, p. 132 (5 février 1764). L’Encyclopédie (Diderot) définit la mélancolie comme « le sentiment naturel de notre imperfection ». Voir G. Minois, Histoire du mal de vivre. De la mélancolie à la dépression, Paris, La Martinière, 2003.

${ }^{22}$ Respectivement BNCF, NA 1050 I, vol. XXV, p. 40 (29 novembre 1769) et vol. XIX, p. 92, p. 157 (16 août et 25 octobre 1767). La première expression rappelle l'inscription Libri animae medicatorium que, selon Diodore, le roi d'Egypte Osmanduas avait fait placer au fronton de sa bibliothèque, et que l'on trouve fréquemment citée par Juste-Lipse, auteur assidûment fréquenté par Pelli dans ses « vertes années ».
} 
Le trait n'est pas sans rappeler le vocabulaire de la lecture dévote : semblable en cela aux effets de la foi pour l'âme, le livre est remède aux maux de l'esprit et aux déceptions mondaines.

« On dira que le travail de bureau est presque un doux loisir, mais certaines fois en vérité un agriculteur habitué à labourer son champ souffre moins qu'un savant à se consumer le cerveau sur les livres ${ }^{23}$. Autant que d'une réflexion sur la pathologie des gens de lettres, la remarque témoigne de la manière dont les professions intellectuelles s'imposent dans l'imaginaire social à travers la conscience d'une communauté de pratiques. Alors que la représentation des maux du corps lisant suit chez Pelli l'évolution de la pensée médicale, celle des effets physiologiques et psychologiques de la lecture incorpore des traditions plurielles, médicale, humaniste ou religieuse.

\section{UNE RÉVOLUTION DE LA LECTURE?}

L'exemple de Giuseppe Pelli permet de comprendre comment les pratiques savantes anciennement structurées se trouvent travaillées de l'intérieur par les mutations de la librairie $\mathrm{du} \mathrm{XVIII}{ }^{\mathrm{e}}$ siècle et par l'évolution des fonctions sociales de la lecture. Confronté à l'inflation de la production imprimée, Pelli se lamente sans cesse des mauvais livres qui - topos depuis le XVII ${ }^{\mathrm{e}}$ siècle - noient les bons ouvrages, effacent la mémoire des anciens et poussent la galaxie Gutenberg vers une inexorable révolution. Les manières de lire qu'il met en œuvre fonctionnent comme autant de parades contre cette invasion livresque. Beaucoup d'ouvrages font l'objet d'une lecture rapide, qu'il « regarde à la hâte », «feuillette », " parcourt superficiellement », " voit mécaniquement » ou « en vitesse ». " Aujourd'hui, écrit-il en 1771, je ne lis entièrement que peu de livres, mais j'en feuillette un très grand nombre, et autant que je peux pour voir ce qu'ils contiennent ${ }^{24}$. C'est à une lecture cursive du même genre que Giovanni Lami, le fondateur des Novelle letterarie, doit recourir pour rendre compte chaque semaine à ses lecteurs des nouveautés de librairie ${ }^{25}$. D'autres ouvrages sont abandonnés en cours de route. "Aujourd'hui, confesse en 1770 notre diariste, il n'y a que peu de livres dont je puisse terminer la lecture. La manière dont ils sont écrits, l'argument - petit, sec, inutile, etc., la façon dont il est traité, la trivialité de l'érudition, ou cent autres défauts que je sens en moi-même, font qu'ils me tombent le plus souvent des mains après la lecture de quelques pages, et que je les abandonne $»^{26}$. Le chemin du lecteur est ainsi jonché de nombreuses épaves, débris ineptes et boursouflures de la production contemporaine. A plusieurs reprises enfin, Pelli avoue ne plus savoir lire longuement : il en accuse les devoirs qui l'assaillent, la vieillesse qui l'émousse, mais surtout ces livres qui lui déplaisent, le fatiguent, l'endorment ou se montrent incapables de retenir sa pensée ${ }^{27}$. Dans leur diversité, ces comportements vagabonds appartiennent bien à la figure de la lecture "extensive », portée par la multiplication et la diversification de la production imprimée. Mais ces manières de lire se trouvent déjà dans la boîte à outils des bibliographes et des compilateurs de la première modernité, infatigables bâtisseurs de bibliothèques sans murs. Derrière elles se profile encore l'idéal humaniste du vagabondage parmi les livres. «D'une chose je passe facilement à une autre, pour y retourner à nouveau après quelque temps $»$ : identifier dans cette flânerie l'image

\footnotetext{
${ }^{23}$ Ibid., vol. III, p. 72, $1^{\text {er }}$ septembre 1760 . D. Roche relève des remarques similaires chez les lettrés français («L'intellectuel au travail », art. cit., p. 476).

${ }^{24}$ BNCF, NA 1050 I, vol. XXVII, p. 182, 3 juillet 1771.

${ }^{25}$ B. Dooley, «La seconde révolution de la lecture dans l'Italie du XVIII ${ }^{\mathrm{e}}$ siècle », Revue d'histoire moderne et contemporaine, $49, \mathrm{n}^{\circ} 3,2002$, p. 69-88, p. 77-78.

${ }^{26}$ BNCF, NA 1050 I, vol. XXVII, p. 13, 28 décembre 1770.

${ }^{27}$ Ibid., vol. XIV, p. 26-27 (1 ${ }^{\text {er }}$ avril 1765) ; vol. XI, p. 129 (2 février 1764) ; vol. XXVIII, p. 23 (8 août 1771).
} 
d'un nouveau style de lecture, superficiel et désinvolte, serait sous-estimer la force de l'héritage d'un Montaigne dans les pratiques savantes du XVIII ${ }^{\mathrm{e}}$ siècle $^{28}$.

L'afflux des livres et l'«extensivité » du lire ne font pas de la lecture une activité anodine. Elle reste le filtre qui structure l'expérience, à travers lequel seulement le monde devient accessible et pensable. Aux ouvrages les plus dangereux, Pelli oppose donc une arme suprême, le refus de lecture. C'est le sort infligé par exemple aux Recherches sur l'origine du despotisme oriental de Boulanger (Genève, 1761), ouvrage impie qu'il tient entre ses mains mais ne lit pas «pour ne pas perdre de temps ${ }^{29}$. L'évocation des lectures romanesques, auxquelles les historiens du livre ont prêté un rôle central dans l'évolution des manières de lire à la fin du XVIII ${ }^{\mathrm{e}}$ siècle, met en évidence la manière dont le lettré intègre ce genre dans une pratique réfléchie et réflexive de la lecture, conservant tout son efficace. Quoiqu'il dise porter peu d'estime aux romans, Pelli en est comme nombre de ses contemporain(e)s un lecteur infatigable, enthousiaste et sensible, dévorant Voltaire, Marmontel, Sterne ou Richardson. Il se raconte littéralement happé par la Princesse de Babylone, que malgré une journée fatigante il ne peut s'empêcher de finir avant de se mettre au lit ${ }^{30}$. Les longs comptes rendus qu'il donne de ces lectures éclairent le rapport de Pelli au texte romanesque. Plus que pour son intrigue (résumée en quelques lignes), le roman vaut comme un recueil de maximes ou d'images du monde contemporain, dont Pelli s'attarde à dresser le florilège. "L'œuvre est en elle-même obscène, écrit-il des Bijoux indiscrets de Diderot, et à ne pas mettre entre toutes les mains, mais je dois confesser que pour connaître le monde elle a quelque valeur, et qu'elle contient une critique délicate et des allusions fines aux mœurs françaises ${ }^{31}$. Ce régime de lecture, assez courant au XVIII ${ }^{\mathrm{e}}$ siècle $^{32}$, se modifie sous l'effet de textes qui éduquent leur public à lire autrement. Il semble ainsi que les contes de Voltaire aient insensiblement appris à Pelli à apprécier pour eux-mêmes la verve romanesque et les ressorts narratifs, malgré l'invraisemblance des intrigues, les attaques contre la religion, et «bien qu'ils n'édifient pas, et n'enseignent pas grand-chose $»^{33}$. La lecture des pièces dramatiques, faite dans l'intimité ou récitée en petit comité, donne à voir un troisième rapport au texte, marqué par une plus grande « fluidité affective » (C. Labrosse) entre les personnages et le lecteur, voire entre les lecteurs de l'œuvre. La lecture de la Fille naturelle de Rétif de la Bretonne émeut ainsi profondément Giuseppe Pelli, parce qu'elle lui donne à revivre sa propre expérience de père adoptif. Sa manière de lire confine dans ce cas à la lecture sentimentale du préromantisme, qui postule l'émotion du lecteur comme condition de la correcte réception du texte ${ }^{34}$.

Loin d'abandonner la lecture intensive, le lecteur aménage donc au cœur même de la tourmente livresque les conditions d'une lecture assidue et réfléchie. Au fil des années, Pelli tisse même avec certains livres des relations d'une rare intensité. Il a trente ans lorsqu'il lit pour la première fois les Essais de Montaigne, dans l'édition en 10 volumes in- $12^{\circ}$ annotée par P. Coste (Londres, 1754). Le style lui paraît d'abord un peu obscur, « mais avec l'usage il me deviendra plus intelligible », se convainc-t-il. Cette lecture est une véritable révélation, qui

\footnotetext{
${ }^{28}$ «Là je feuillette à cette heure un livre, à cette heure un autre, sans ordre et sans dessein, à pièces décousues : Tantôt je rêve, tantôt j'enregistre et dicte, en me promenant, mes songes que voici » (Montaigne, Essais, livre III, chap. 3).

${ }^{29}$ BNCF, NA 1050 I, vol. VII, p. 68-69, 10 avril 1762.

${ }^{30}$ Ibid., vol. XXI, p. 179-181, 24 juillet 1768. Il s'agit du conte de Voltaire publié en 1768.

${ }^{31}$ Ibid., vol. VII, p. 33-35, 5 mars 1762.

${ }^{32}$ C. Labrosse, Lire au XVIII e siècle. La Nouvelle Héloüse et ses lecteurs, Lyon, PUL/CNRS, 1985, p. 39-40.

${ }^{33}$ BNCF, NA 1050 I, vol. XXI, p. 181, 24 juillet 1768.

34 « Ma situation présente m'a fait goûter ce roman certainement plus que je l'aurais goûté en d'autres temps... Des situations intéressantes, des sentiments égaux aux miens ont réveillé toute ma sensibilité, et j'ai trouvé que je me suis dit à moi-même ce que se disait d'Azinval en cette nuit agréable décrite p. 19 et suiv. T. I, quand il eut recueilli la jeune Marion » (ibid., série II, vol. X, f. 1759v-1760r, 26 février 1782, cité par R. Pasta, « Profilo di un lettore », art. cit., p. 219). Sur la lecture préromantique, A. Montandon, « Le lecteur sentimental de JeanPaul », in Id. (dir.) Le lecteur et la lecture dans l'œuvre, Clermont-Ferrand, 1982, p. 25-33.
} 
irrigue ensuite toute l'écriture des Efemeridi. A la confrontation prudente, puis au relevé des similitudes entre le caractère de Montaigne et le sien, succède une connivence sans cesse approfondie, abolissant la distance temporelle et l'écart social ${ }^{35}$. Au fil des volumes ensuite, le dialogue entre les Efemeridi et les Essais se déplace de la question de la connaissance de soi à celle de l'écriture de soi. Plus que n'importe quelle autre, la lecture de Montaigne est une maïeutique, qui amène jusqu'à l'expression écrite les sensations confuses de l'expérience individuelle : «si je cite souvent Montaigne, c'est que je trouve en lui beaucoup de choses qui me conviennent, parce qu'il me permet de développer de nombreuses idées que j'ai confusément et qu'il me met en état de raisonner sur les sujets qu'il traite, plus précisément que ce que j'aurais pu faire avant de le rencontrer $\gg{ }^{36}$.

Ce commerce intense et familier du texte de Montaigne vient rappeler, jusque dans ses aspects matériels, la rumination puritaine ou cléricale de la Bible. Pelli fait sien le mot du cardinal du Perron qui qualifiait les Essais de «bréviaire des honnêtes gens » ${ }^{37}$. Il le porte presque toujours dans sa poche, le tient à portée de main sur son bureau, se plaisant en sa compagnie, l'ouvrant de temps en temps, pour une page ou deux, comme on lit la Bible ou comme on consulte un ami. Chemin faisant, le rapport affectif entretenu avec l'objet livre se déplace en effet vers l'homme, dans un mouvement qui n'est pas sans rappeler certains lecteurs de Rousseau ${ }^{38}$. Pelli baptise sa chienne Gournay, du nom de la « fille d'alliance » de Montaigne, fait sienne la devise «Que sais-je ? » et porte au doigt, gravé sur une agate orientale, le portrait de celui qu'il n'appelle presque plus, à partir de 1761, que «mon (cher) Montaigne ». La lecture des Essais imprime enfin sa marque, on l'a vu, sur la manière qu'a Pelli de vivre avec les livres: le plaisir éprouvé à retarder une lecture attendue, le vagabondage parmi les livres, l'annotation liminaire des ouvrages, la représentation mentale du cabinet de travail inscrivent l'héritage humaniste au cœur des pratiques du dernier XVIII ${ }^{\mathrm{e}}$ siècle.

«Toute analyse historique de cette entreprise complexe et protéiforme qu'est la lecture doit résister aux sirènes de la grande théorie et à la tentation de distinguer des changements tranchés, pour accepter la liberté du paradoxe et de la contradiction ${ }^{39}$. Trois caractères semblent finalement individualiser la révolution du lire chez ces lecteurs caméléons que sont les professions intellectuelles : la réactivation et le renforcement de modalités anciennes de la lecture extensive, l'enrichissement (préromantique) des figures traditionnelles (religieuse, érudite, humaniste) de la lecture intensive, le métissage médical et philosophique des imaginaires du corps lisant.

\footnotetext{
${ }^{35}$ Il évoque la «manière uniforme de penser [qui] unit nos âmes d'un lien fort » (BNCF, NA 1050 I, vol. II, p. 191, 12 juin 1760), mais relève tout de même la dissemblance des goûts et des coutumes, « parce que je ne vis pas au XVI ${ }^{\mathrm{e}}$ siècle, parce que je ne suis pas gascon, ou homme de guerre » (vol. XXIX, p. 118, 7 juillet 1772).

${ }^{36}$ Ibid., vol. V, p. 141-142, 9 août 1761.

${ }^{37}$ Ibid., vol. VI, p. 2-3, 20 septembre 1761 et vol. XXVIII, p. 103, 11 novembre 1771.

${ }^{38}$ R. Darnton, «La lecture rousseauiste et un lecteur « ordinaire » au XVIII ${ }^{\mathrm{e}}$ siècle », in R. Chartier (dir.), Pratiques de la lecture, op. cit., p. 167-208.

${ }^{39}$ A. Grafton, «Le lecteur humaniste », in G. Cavallo, R. Chartier (dir.), Histoire de la lecture dans le monde occidental, Paris, Seuil, 1997, p. 213.
} 